\title{
tic\&société
}

Vol. 11, $\mathbf{N}^{\circ} 1$ | 2ème semestre 2017

L'éducation critique aux médias à l'épreuve du numérique

\section{Pour une approche de l'éducation critique aux médias par le décryptage des logiques politiques, économiques, idéologiques et éditoriales du numérique}

\section{Sophie JEHEL et Alexandra SAEMMER}

\section{OpenEdition Journals}

\section{Édition électronique}

URL : http://journals.openedition.org/ticetsociete/2251

DOI : 10.4000/ticetsociete.2251

Éditeur

Association ARTIC

Édition imprimée

Pagination : 47-83

\section{Référence électronique}

Sophie JEHEL et Alexandra SAEMMER, «Pour une approche de l'éducation critique aux médias par le décryptage des logiques politiques, économiques, idéologiques et éditoriales du numérique », tic\&société [En ligne], Vol. 11, № 1 | 2ème semestre 2017, mis en ligne le 01 septembre 2017, consulté le 14 novembre 2019. URL : http://journals.openedition.org/ticetsociete/2251 ; DOI 10.4000/ticetsociete.2251 
tic\&société - 11(1), 2017

\section{Pour une approche de l'éducation critique aux médias par le décryptage des logiques politiques, économiques, idéologiques et éditoriales du numérique}

\section{Sophie JEHEL}

Sophie Jehel est maîtresse de conférences en sciences de l'information et de la communication à l'Université Paris 8, agrégée en sciences économiques et sociales, chercheure au Laboratoire CEMTI (Centre d'étude sur les médias, les technologies et l'internationalisation), responsable du parcours Web et cultures participatives du master Industries culturelles et créatives. Ses recherches portent sur le rôle des médias dans la socialisation, les pratiques médiatiques des jeunes, l'éducation aux médias, mais aussi sur la déontologie et la régulation des médias. Elle a publié Stéréotypes, discriminations et éducation aux médias (L'Harmattan, 2016) avec Laurence Corroy, dans la continuité du projet européen eEngagement Against Violence.sophie.jehel@univ-paris8.fr

\section{Alexandra SAEMMER}

Alexandra Saemmer est professeure des universités en sciences de l'information et de la communication (laboratoire CEMTI, Université Paris 8). Ses recherches portent sur la production du sens dans le texte numérique (presse en ligne, publicité, e-albums, littérature numérique, réseaux sociaux...). Les formes et les figures du texte numérique sont étudiées dans une approche relevant de la sémiotique sociale, qui s'intéresse tout autant aux matérialités de la communication et aux stratégies discursives qu'aux représentations individuelles et partagées guidant la réception. Ses plus récentes monographies sont Rhétorique du texte numérique (Presses de l'Enssib, 2015) et l'ouvrage collectif, coédité avec Nolwenn Tréhondart, Livres d'art numériques, de la conception à la réception (Hermann, 2017). alexandra.saemmer@gmail.com 


\title{
Pour une approche de l'éducation critique aux médias par le décryptage des logiques politiques, économiques, idéologiques et éditoriales du numérique
}

\begin{abstract}
Résumé : Nous proposons dans cet article une grille d'interprétation nourrie de trois approches facilitant l'accès à la complexité des enjeux politiques, économiques, idéologiques et éditoriaux des médias numériques: l'économie politique, la sociologie des usages et de la réception, et la sémiotique sociale. Nous montrons que ces trois approches, jusqu'alors rarement convoquées de façon conjointe, constituent une démarche efficace pour expliciter le fonctionnement des industries culturelles et médiatiques, pour décrypter les utopies et les discours idéologiques agissant sur les usages et les pratiques de réception, mais aussi pour encourager des pratiques de résistance, voire d'émancipation vis-à-vis de leurs logiques de domination.
\end{abstract}

Mots-clés : économie politique, sociologie des usages, études de réception, sémiotique sociale, décryptage, idéologies.

\begin{abstract}
In this article, we present an interpretive grid based on three ways to approach the complex issues raised by digital media: political economy, sociology of uses and reception, and social semiotics. We show how combining these approaches, rarely employed together, may offer an effective means of explaining the functioning of cultural and media industries, of deciphering utopic and ideological discourses that act on practices of media reception and use, but also of encouraging practices of resistance or even emancipation against dominant power structures.
\end{abstract}

Keywords: political economy, sociology of uses and reception, social semiotics, decryption, ideologies.

Resumen: Este artículo propone un modelo de interpretación basado en tres enfoques que facilita el análisis de la 
Pour une approche de l'éducation critique aux médias par le décryptage des logiques politiques, économiques, idéologiques et éditoriales du numérique

complejidad de las implicaciones políticas, económicas, ideológicas y editoriales de los medios digitales: la economía política, la sociología de los usos y de la recepción, y la semiótica social. Muestra que estos tres enfoques, hasta ahora escasamente utilizados al mismo tiempo, no sólo constituyen un método eficaz para investigar el funcionamiento de las industrias culturales y mediáticas, sino que sirven también para analizar las utopías y los discursos ideológicos sobre los usos y las prácticas de recepción, así como también para fomentar las prácticas de resistencia, e incluso emancipatorias, frente a las lógicas de dominación.

Palabras clave: economía política, sociología de los usos, estudio de la recepción, semiótica social, descifrado, ideologías. 


\section{Introduction}

L'éducation aux médias est depuis ses origines associée à la construction d'un esprit critique et conçue comme une "préparation des citoyens à l'exercice de leurs responsabilités ", pour reprendre les termes de la déclaration de Grunwald publiée sous l'égide de I'UNESCO (1982). La notion d'esprit critique reste cependant assez floue, celle d'éducation aux médias également (Gonnet, 2001). Nous proposerons, dans cet article, une approche ${ }^{1}$ reposant sur l'analyse des logiques politiques, économiques et idéologiques à l'œuvre dans le fonctionnement des industries du numérique, héritières de l'industrie culturelle des années 1920, à partir des recherches développées en sciences de l'information et de la communication (SIC) et dans les sciences économiques et sociales. Nous montrerons comment cette approche peut, en facilitant l'accès à la complexité de l'entremêlement des enjeux politiques, économiques, idéologiques, éditoriaux et discursifs des industries du numérique, construire des approches pédagogiques susceptibles de transformer le regard des apprenants sur les contenus et les dispositifs médiatiques.

L'introduction du numérique dans l'école soulève un certain nombre de questions et suscite des résistances. Nous commencerons par l'analyse des politiques éducatives menées en France depuis 2013 pour l'illustrer. Le cas français, au-delà de ses spécificités, et notamment du rôle historique de l'école dans la diffusion du modèle républicain, met en lumière l'immixtion de préoccupations politiques et industrielles au sein des institutions éducatives, qui peuvent entrer en contradiction avec le projet d'émancipation de l'école. Ce mélange des genres se rencontre dans d'autres contextes, mais a acquis, en France, un caractère hautement paradoxal. Nous proposons de démontrer que les SIC peuvent délivrer, face à cette situation, des clés d'interprétation d'une importance cruciale pour les enseignants et les éducateurs d'aujourd'hui. La compréhension du fonctionnement des industries du numérique, en particulier des grandes plateformes transnationales qui sont en train de

\footnotetext{
${ }^{1}$ Approche que nous mettons également à l'épreuve dans un séminaire que nous avons ouvert en 2015 au Cemti (Centre d'études sur les médias, les technologies et l'internationalisation), à l'université Paris 8 en France.
} 
Pour une approche de l'éducation critique aux médias par le décryptage des logiques politiques, économiques, idéologiques et éditoriales du numérique

s'approprier et de fermer le Web (Anderson et Wolff, 2010), devrait être un préalable à la mise en place de pédagogies numériques.

\section{Détacher l'éducation aux médias et à l'information des injonctions politiques paradoxales qui la traversent : le cas français}

En France, la récente loi pour la refondation de l'école ${ }^{2}$ a donné à l'éducation aux médias et à l'information (EMI) une nouvelle impulsion. Selon le rapport officiel publié en annexe de la loi,

il est impératif de former les élèves à la maîtrise, avec un esprit critique, de ces outils qu'ils utilisent chaque jour dans leurs études et leurs loisirs et de permettre aux futurs citoyens de trouver leur place dans une société dont l'environnement technologique est amené à évoluer de plus en plus rapidement.

Or les conditions de sa mise en œuvre restent discutables, centrées sur les outils bien plus que sur la formation des enseignants, malgré son niveau notoirement insuffisant (FrauMeigs et al., 2013).

\subsection{Une polarisation des dotations financières sur les équipements et une conception fonctionnaliste $d u$ numérique}

La politique éducative récente en France a renforcé l'urgence d'une réflexion sur l'accompagnement des enseignants, dans ce moment clé où l'école est censée devenir une "école numérique " pour penser les exigences d'une éducation critique aux médias en contexte numérique. Des mesures symboliques, par exemple la reconnaissance dans la loi de l'éducation aux médias et à l'information comme l'une des missions de l'école, présupposent l'aménagement d'espaces de réflexion sur l'EMI. Certaines de ces mesures accélèrent

\footnotetext{
${ }^{2}$ Loi d'orientation et de programmation pour la refondation de l'École de la République $n^{\circ} 2013-595$ du 8 juillet 2013.
} 
pourtant surtout l'introduction des structures médiatiques et des industries du numérique dans l'école.

Le grand "plan numérique pour l'école " (2015-2018) repose sur la dotation d'un milliard d'euros. II s'accompagne de mesures administratives, comme la restructuration des services de l'Éducation nationale liés à l'EMI (intégration du Clemi dans Canopé en décembre 2015), et de liens de partenariat renforcé avec les professionnels des médias, en particulier FranceTv Éducation, service de France Télévisions, mais aussi la plateforme Mediaeducation.fr (mars 2016). Cette politique éducative est marquée par une polarisation forte sur la question des équipements et des outils. C'est par exemple grâce aux tablettes que "l'école numérique » est censée se réaliser,

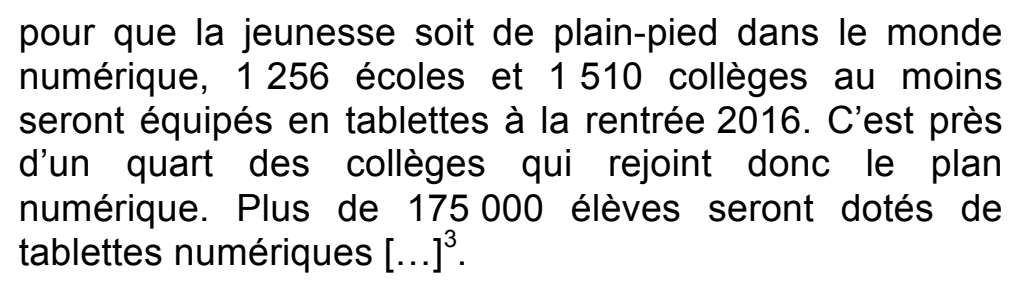

Le partenariat avec la firme transnationale Microsoft pour l'équipement en logiciels a été renforcé, avec un volet sur la formation des acteurs, des cadres et des enseignants, aux usages des logiciels. Sont également favorisées l'expérimentation de plateformes de jeux sérieux et la mise en place de supports numériques (convention du 30 novembre 2015). La plateforme FUN de MOOC est lancée en 2013. L'EMI risque alors de se dissoudre dans une éducation au numérique et par le numérique, prenant la forme d'un accompagnement à la prise en main d'outils dans une perspective fonctionnaliste, afin de résoudre diverses défaillances du système éducatif qui ont leurs racines ailleurs.

Pourtant, la définition de l'éducation à la citoyenneté, qui est l'un des axes complémentaires de l'éducation aux médias, laisse voir que les responsables de l'Éducation nationale sont conscients des enjeux bien plus larges que représente le travail pédagogique sur les contenus et les dispositifs issus des industries médiatiques :

\footnotetext{
${ }^{3}$ Selon le site officiel du ministère (http://www.gouvernement.fr/action/l-ecolenumerique), s'inspirant du discours de François Hollande du 7 mai 2015.
} 
Pour une approche de l'éducation critique aux médias par le décryptage des logiques politiques, économiques, idéologiques et éditoriales du numérique

\begin{abstract}
L'école doit donner aux élèves les instruments de leur autonomie et de leur responsabilité, en leur enseignant la distance intellectuelle, en développant leur esprit critique, en leur apprenant les règles d'un bon usage des moyens d'information et de communication, en leur transmettant les valeurs, les concepts et les outils propres à garantir leur propre protection en même temps que le respect d'autrui. II s'agit avant tout de leur faire prendre conscience que les conditions de production de l'information, le support ou le canal de diffusion ne sont pas neutres: qu'ils conditionnent la forme des messages, induisent une série de choix et donc surdéterminent leur contenu (Becchetti-Bizot et Brunet 2007, pp. 18-19).
\end{abstract}

\title{
1.2 Les trois paradoxes de cette politique éducative au numérique
}

Un premier paradoxe apparaît lorsqu'on rappelle la signification première du mot skholè. En grec ancien, cette notion désigne un arrêt des occupations liées à la subsistance, une suspension de l'affairement frénétique de la vie quotidienne marquée par son lot d'asservissements. Or, quand il s'agit de " faire entrer le numérique à l'école », la motivation semble souvent être au contraire d'acculturer l'apprenant le plus tôt possible aux occupations liées à la subsistance et à l'employabilité. L'enjeu pédagogique est mis au service d'objectifs économiques, qui, au-delà de l'employabilité, sont ceux du développement de filières industrielles. Un rapport de 2013 sur la « structuration de la filière du numérique éducatif : un enjeu pédagogique et industriel», auquel a participé l'inspection générale de l'Éducation nationale, défend explicitement une stratégie de soutien à ces industries. II s'agit de promouvoir la création d'emplois dans cette filière, que le plan numérique pour l'école prévoit également de soutenir. Dans les appels d'offres les plus récents, comme l'appel Services innovants numériques Éduthèque (Siné) en 2017, l'objectif annoncé est ainsi de "développer des services innovants pour l'école et [de] réaliser des contenus enrichis par des établissements publics de référence et leurs prestataires de la French Tech » (p. 6), démontrant l'imbrication étroite entre les visées pédagogique et industrielle de ces services. 
Le second paradoxe apparaît quand on compare la place de l'éducation aux médias dans les curricula et la liste toujours plus longue des objectifs politiques assignés ou accolés à cet enseignement: la lutte contre les inégalités sociales et de genre, la lutte contre le harcèlement scolaire et le cyberharcèlement, la prévention des phénomènes de radicalisation, la lutte contre les discours "complotistes ", la lutte contre l'hyperconnexion, la démystification des outils courants imposés par l'industrie culturelle du numérique et du divertissement... Cette éducation reste en effet le parent pauvre d'un système éducatif français centré sur les "fondamentaux », compris comme des savoirs disciplinaires neutres, ce qui fait dire à François Rastier (2013) qu'elle serait une "pseudo-matière » (p. 70), au risque de renforcer les dispositions traditionnellement hostiles d'une partie du corps enseignant vis-à-vis de l'introduction des médias à l'école.

Le troisième paradoxe consiste à promouvoir le numérique à l'école pour rattraper le retard pris par la France dans le classement Pisa, alors même que le rapport Pisa de 2015 concluait à une "incidence mitigée " de l'utilisation des nouvelles technologies en classe, "dans le meilleur des cas". Dans ses résultats, le bénéfice pour les "élèves utilisant modérément les ordinateurs " semble léger, tandis que ceux « utilisant très souvent les ordinateurs à l'école obtiennent des résultats bien inférieurs dans la plupart des domaines d'apprentissage, même après contrôle de leurs caractéristiques sociodémographiques " (OCDE, 2015, p. 1). Au moment même où la France consentait un important investissement dans les tablettes, l'OCDE constatait que les pays qui avaient suivi ce genre de politique n'avaient vu " aucune amélioration notable des résultats de leurs élèves en compréhension de l'écrit, en mathématiques et en sciences " et que l'usage des TIC ne semblait pas résorber «les écarts de compétences entre élèves favorisés et défavorisés » (ibid., p. 1). L'étude concluait sur le rôle déterminant de "l'acquisition par chaque enfant d'un niveau de compétences de base en compréhension de l'écrit et en mathématiques" pour lutter contre les inégalités et envisageait le lien probable entre "le développement d'une compréhension conceptuelle et d'une réflexion approfondies » et " des interactions intensives entre enseignants et élèves un engagement humain précieux duquel la technologie peut parfois nous détourner » (ibid. p. 4). 
Pour une approche de l'éducation critique aux médias par le décryptage des logiques politiques, économiques, idéologiques et éditoriales du numérique

\subsection{Un projet qui manque de cohérence}

Le choix de privilégier l'attention portée aux équipements sur les questions de formation des enseignants et le contenu des enseignements est sans aucun doute la marque de la pression économique qui traverse le champ éducatif, plus intense en contexte de croissance végétative et de chômage de masse. Cette orientation n'est pas nouvelle. Pierre Moeglin (2015), observateur des politiques et des industries éducatives, constatait, en 2015 : «Plan après plan, le numérique à l'école commet les mêmes erreurs. Malgré tous les avertissements. En toile de fond, une OPA de l'approche productiviste en éducation aux dépens de l'approche culturelle » (pp. 62-63). C'est cependant un choix qui s'inscrit dans l'ignorance des nombreux travaux de chercheurs qui montrent que les inégalités sociales face au numérique sont d'ordre cognitif bien plus que d'équipement (Bourdeloie, 2012 ; Brotcorne et Valenduc, 2009 ; Hargittai, 2002).

Les incohérences de ces politiques sont nombreuses: équiper en tablette des adolescents dont on souhaiterait aussi qu'ils s'initient au code, alors que la tablette est un dispositif particulièrement fermé qui ne facilite aucunement l'accès au code et à sa modification; conforter le monopole de Microsoft dans l'école tout en promettant, par le biais d'appels d'offres faisant appel à la French $T e c h$, le développement d'une filière éducative nationale ; prévoir la signature d'une charte sur la vie privée sans garantir la maîtrise des algorithmes de traçage et de récolte de données par les acteurs de l'école (Agacinski, 2016). Cette politique continue alors à poursuivre des objectifs "flous », différents selon les acteurs (poids du cartable, inégalités sociales, rattrapage du retard scolaire, différenciation de l'enseignement...), sans l'inscrire, au-delà des symboles, dans un projet clair pour l'école (Moeglin, 2015). Le rôle politique majeur de l'école en France, depuis son enracinement dans la $3^{\mathrm{e}}$ République, exigerait cependant une attention plus grande aux objectifs de cohésion sociale et aux fractures cognitives dont les signes se manifestent de plus en plus. Audelà du cas français, c'est la question de la capacité de l'école à échapper aux logiques présentistes, fonctionnalistes, et à inscrire l'EMI dans ses objectifs humanistes, en cohérence avec son rôle de pont entre le passé et le présent, pour 
transmettre aux jeunes générations le souci de la fragilité du monde et sa compréhension (Arendt, 1972).

\section{Trois approches méthodologiques pour l'EMI}

Nous chercherons, dans cette contribution, à redessiner les contours de l'éducation aux médias en contexte numérique en mettant au centre la nécessité d'une approche critique des logiques politiques et économiques du numérique, l'analyse de l'incarnation de ces logiques dans l'épaisseur sémiotique des artefacts médiatiques qui organise l'éditorialisation des présences numériques et oriente les pratiques de réception, mais aussi une sensibilisation aux lignes de fuite et aux poches de résistance toujours possibles.

Nous proposons une grille d'interprétation du numérique articulant trois approches: l'économie politique des médias, la sociologie des usages et de la réception, la sémiotique sociale des interfaces. Ces trois approches, nourries des résultats de la recherche en SIC et en sciences sociales, mais rarement mobilisées de façon conjointe, permettent de construire une démarche efficace pour expliciter le fonctionnement des industries culturelles et médiatiques, pour permettre le décryptage des utopies et des discours idéologiques agissant sur les pratiques de réception, mais aussi pour ouvrir des espaces de liberté.

Les médias sont un terme polysémique qui désigne des objets complexes. Rémy Rieffel (2005) distingue quatre niveaux d'analyse : ils

doivent être conçus[, dans un premier temps,] comme un ensemble de techniques de production et de transmission de messages à l'aide d'un canal, d'un support (journal papier, ondes hertziennes, câble, etc.) vers un terminal (récepteur-écran), ainsi que comme le produit de cette technique (journaux, livres, émissions) ; dans un second temps, comme une organisation économique, sociale et symbolique avec ses modalités de fonctionnement, ses acteurs multiples, qui traite ces messages et qui donne lieu à des usages variés (p. 31).

Nous privilégierons ici l'analyse de l'organisation économique, sociale et symbolique des médias, et nous 
Pour une approche de l'éducation critique aux médias par le décryptage des logiques politiques, économiques, idéologiques et éditoriales du numérique

procéderons d'abord à partir de l'économie politique des médias. Celle-ci permet à la fois de situer les industries du numérique dans le prolongement de l'industrie culturelle et d'insister sur leur rôle dans le développement du « nouvel esprit du capitalisme " (Boltanski et Chiapello, 1999) et la diffusion de l'idéologie néolibérale (Bouquillion et Matthews, 2010 ; Dardot et Laval, 2009; Rebillard, 2007). Dans cette approche, les industries du numérique sont autant les symboles d'une idéologie néolibérale qui promeut l'extension des logiques du marché à toutes les sphères de la vie privée, la disparition des hiérarchies, la mise en avant de la responsabilité individuelle, que les outils de nouvelles modalités de production et de récupération de la valeur que décrivent les théoriciens du digital labor (Casilli et Cardon, 2015; Scholz, 2012). Dans le prolongement de la théorie de l'industrie culturelle, cette approche invite à un premier niveau d'introspection idéologique en s'intéressant aux contextes économiques de production des médias et en engageant une critique des contraintes que les enjeux marchands exercent sur les processus de conception, d'édition, de diffusion et de réception.

La question des usages et de la réception des médias est également cruciale dans le contexte de l'EMI, puisque l'intervention de l'école pour promouvoir un usage autonome et responsable des médias présuppose une compréhension des usages spontanés des adolescents, en particulier de la manière dont le numérique reconfigure les inégalités sociales. Cette analyse s'appuiera sur la sociologie des usages et sur la sociologie de la réception, dont les résultats peuvent être mobilisés dans l'approche sémiotique des interfaces.

Cette approche sémiotique se penche d'une part sur les éléments sociaux qui guident le processus d'usage et de réception d'un artefact médiatique, les représentations individuelles et socialement partagées mobilisées lors de l'usage, d'autre part sur les structures matérielles de l'artefact qui relancent ces représentations ou les mettent au défi. Loin des "outils de décryptage " et des autres grilles de lecture toutes faites parfois mobilisés par la sémiologie " appliquée » à l'EMI, la sémiotique sociale (Saemmer, 2017) n'essaie donc pas de déterminer ce qu'une production médiatique signifie, mais se penche sur le processus d'interprétation lui-même, visant la conscientisation de celui-ci. Elle rejoint ainsi les 
préoccupations historiques des branches critiques de la sémiotique, initiées entre autres par Roland Barthes et Umberto Eco.

Dans son célèbre article consacré à la rhétorique de l'image (1964), Roland Barthes propose une analyse des stéréotypes mobilisés par l'image publicitaire, mais - et cet aspect est souvent oublié - il problématise aussi le « savoir culturel » qui intervient dans l'interprétation de ces stéréotypes. II fait ainsi un premier pas vers une sémiotique sociale, en spécifiant que le sens n'est pas inhérent à un artefact médiatique, mais coconstruit entre les signes et le récepteur, invitant l'interprète à une autoréflexivité qui nous paraît fondamentale dans l'EMI. Une analyse sémiotique d'une image, d'une émission, d'un site Web ou d'une plateforme consiste donc à circonscrire avec le plus de précision possible les éléments qui pèsent sur les processus interprétatifs, les usages et la réception, sur les plans individuel et collectif. Cette approche, fondée sur la constitution de "communautés interprétatives " au sein de la classe, permet de se déprendre d'une posture magistrale et insère les jeunes apprenants dans le processus interprétatif luimême en faisant émerger les éléments multiples, sociaux, éditoriaux et discursifs qui guident ce processus.

II s'agit d'identifier, par un travail d'interprétatif collectif, comment les pratiques de conception et de réception sont modélisées par les caractéristiques matérielles d'une production médiatique : son interface, son design, l'agencement des signes, son "architexte " logiciel et algorithmique (les cadres et formats qui, en contexte numérique, régissent la forme et l'agencement des signes sans être toujours percevables à l'écran [Jeanneret et Souchier, 1998]). Ces modélisations de pratiques reflètent jusqu'à un certain point les représentations, les motivations et les "allant de soi » des concepteurs et des producteurs de l'artefact, leurs stratégies éditoriales, économiques et politiques. Les usages épousent ces modélisations jusqu'à un certain point et, parfois, en divergent. Ces convergences et divergences dépendent en outre des représentations, des motivations et des "allants de soi » que les publics mobilisent à leur tour, face à la production médiatique ainsi que par leur adhésion plus ou moins inconditionnelle aux discours d'accompagnement idéologiques mobilisés par les fabricants des plateformes. 
Pour une approche de l'éducation critique aux médias par le décryptage des logiques politiques, économiques, idéologiques et éditoriales du numérique

Le concept d'interprétant proposé par Charles Sanders Pierce permet de conceptualiser l'imbrication dynamique entre les représentations individuelles et collectives et les caractéristiques matérielles de l'artefact, lors de l'usage et de la réception d'une production médiatique. L'interprétant désigne d'abord l'idée qu'un signe fait naître chez le récepteur. Cette idée est elle-même un signe, ouvrant ainsi le processus dynamique de la sémiose (Pierce, 1987). Or le récepteur ne peut librement prendre sa décision sémiotique et interpréter les signes à sa guise. Une idée suscitée par un signe peut, du moins de façon temporaire, recevoir "le flambeau de la vérité " (Pierce, 1895, parag. 339), et cette décision de vérité a des motivations diverses, individuelles ou partagées. Robert Marty (2007) rapproche donc l'interprétant de la notion de norme sociale, d'habitus collectif. L'interprétant collectif (Boutaud et Veron, 2007) désigne des représentations partagées dans une société, y compris celles des a priori idéologiques soutenus par des systèmes de domination politiques et économiques. La sémiotique sociale circonscrit ces "noyaux d'appartenance " en mobilisant, notamment, des méthodologies de la sociologie des usages et étudie leur rôle dans les processus de réception des artefacts médiatiques. Mythocritique dans la tradition de Roland Barthes, cette méthodologie tente ainsi d'analyser, comme l'a formulé Umberto Eco (1975),

les connexions secrètes et cachées d'un système culturel donné, les modalités dans lesquelles le travail de production des signes peut respecter ou trahir la complexité de ce réticule sémantique, en le rendant adéquat (ou en le séparant du) travail humain de transformation des états du monde (p. 370).

\section{Comprendre l'emprise de l'économie politique des plateformes sur les contenus médiatiques et numériques}

Dans cette partie, nous souhaitons mettre en évidence l'impact que la structure industrielle des médias et les modèles économiques sur lesquels reposent les grandes plateformes du numérique peuvent avoir sur l'EMI, pour prendre conscience de l'ampleur de l'emprise des modèles économiques sur le 
formatage des contenus et les modalités qu'il peut prendre. II ne s'agit pas, ici, de brosser à grand trait une économie politique des médias, mais de lever le voile sur certains des rouages clés de l'économie des plateformes qui transforment la situation même de l'apprentissage, en faisant de l'école un utilisateur comme un autre des plateformes commerciales, ou qui « en-forment » (Jeanneret, 2016) les contenus sur lesquels elle souhaite travailler.

\subsection{La course à l'audience " maxima » et aux ressources publicitaires}

L'industrie culturelle place historiquement la course à l'audience au cœur de son modèle économique. Dans une logique commerciale, il est indispensable que le chiffre d'affaires réalisé devance l'ensemble des coûts de production. La spécificité des industries culturelles vient de la nécessité de la diffusion de ses produits auprès d'un public de masse permettant de réaliser des gains de productivité, malgré le coût élevé du prototype. Chaque œuvre produite est alors prise dans une double logique, décrite notamment par Edgar Morin (1961), de standardisation et d'invention. La standardisation permet de limiter le risque industriel que représente la production du stéréotype ; elle conduit à des activités de calibrage et de marketing pour construire le public de l'œuvre. L'invention est la marge de liberté toujours nécessaire, sans laquelle la curiosité du public ne pourrait être stimulée. Le poids de la logique d'industrialisation et de formatage des contenus pour limiter les risques de l'échec public est d'autant plus grand que les capitaux nécessaires à la production sont élevés, par exemple dans le cas du cinéma ou de la télévision (Mairesse et Rochelandet, 2015).

Le modèle économique des médias gratuits, celui du marché à deux versants qui repose principalement sur la publicité, et donc sur la valorisation commerciale de l'audience pour la vente des espaces publicitaires, s'inscrit dans la même logique de standardisation et de stéréotypie. II atteint avec le développement de la téléréalité, genre particulièrement présent 
Pour une approche de l'éducation critique aux médias par le décryptage des logiques politiques, économiques, idéologiques et éditoriales du numérique

sur les chaînes commerciales ${ }^{4}$, des niveaux très élevés de réification des identités qui ne manquent pas de créer chez les adolescents des formes de malaise, au-delà des déclarations de principe sur leur mépris pour ces émissions (Jehel, 2015).

Sans entrer davantage dans la description des modèles économiques des industries culturelles, les procédés de calibrage des contenus médiatiques figurent donc au cœur de leur fonctionnement. Parmi ces procédés, on identifie notamment les stéréotypes, les suites ou sequels, la sérialisation, le "genre " entendu comme une catégorie de contenus créée par l'industrie pour permettre l'identification des goûts du public (autour du policier, de l'horreur, de la romance, de la science-fiction...).

Or l'analyse des stéréotypes médiatiques fait partie des objectifs susceptibles de justifier une démarche en EMI (Jehel et Magis, 2016). C'est ce que nous avons pu expérimenter dans le cadre du projet européen e-Engagement Against Violence, cherchant à identifier les stéréotypes discriminatoires pour "lutter » contre l'adhésion qu'ils peuvent susciter. Dans ce cas, il importe que la démarche pédagogique, sans simplifier à l'excès le processus de production, puisse mettre en évidence les enjeux aussi bien commerciaux qu'idéologiques de l'usage des stéréotypes dans les contenus médiatiques. La stéréotypie est une technique de récit, susceptible de refléter la représentation du monde que souhaitent diffuser les producteurs ainsi que la représentation que les producteurs se font de celle de leur public. Cependant, elle modélise les identités de façon figée à travers la répétition de traits de caractère ou de destins associés à des caractéristiques physiques ou sociales, permettant d'identifier des catégories de la population. La contrainte économique favorise alors la diffusion d'un message idéologique.

La critique de la stéréotypie repose aussi sur la limitation des attentes du public, ce qu'Adorno (1990) a appelé son " infantilisation ». Adorno a dénoncé dès les années 1950 les mécanismes de formatage des œuvres qui construisent une attente du public en "détermin[a]nt les schèmes de

${ }^{4}$ En France, ce dispositif médiatique est particulièrement utilisé par les chaînes privées historiques (M6 et TF1), mais aussi par certaines « nouvelles » chaînes de la télévision numérique terrestre apparues en 2005 (NRJ12, W9, NT1, C8). 
comportement du spectateur » (p. 235). Selon lui, la fiction télévisée, qu'il compare à la culture populaire du $19^{\mathrm{e}}$ siècle, diminuerait l'incertitude du public, en lui donnant la possibilité de "se sentir en terrain sûr » (p. 229). Demander d'identifier les stéréotypes de genre ou ethno-raciaux en se penchant à la fois sur la modélisation des usages et de la réception par les processus narratifs et argumentatifs et leur éditorialisation et sur les interprétations standardisées que ceux-ci suscitent potentiellement devrait inciter les apprenants à l'introspection sur leurs choix culturels et aux effets de cette recherche de sécurité en matière culturelle, venant renforcer les déterminismes sociaux des consommations culturelles. La prise de conscience du lien entre stéréotypie et logique commerciale peut ainsi conduire à la préservation, dans les démarches d'EMI, de la découverte d'espaces non commerciaux, ou moins commerciaux, privilégiant des démarches d'auteur, à côté de l'analyse de l'impact du modèle économique sur les techniques de récit.

\subsection{Un modèle basé sur le recueil des données personnelles et la surveillance généralisée}

La prise de conscience de la dimension inéluctablement commerciale du Web est relativement récente. Dans un rapport de l'OCDE (2007), l'évolution des plateformes d'hébergement de contenu vers une logique de marchandisation était diagnostiquée, comme si se refermait l'espoir d'un Web gratuit, coopératif, investi, avant les années 2000 , de la promesse de constituer une alternative aux logiques des médias traditionnels (Bouquillion et Matthews, 2010). De fait, le fonctionnement des plateformes numériques s'est rapproché de celui des industries médiatiques qui l'ont précédé, tout en inventant de nouvelles formes de financement.

Dans le même rapport, l'OCDE distingue cinq modèles de financement des plateformes d'hébergement de contenu: les donations bénévoles, le modèle de paiement par l'usager, la valorisation d'espaces publicitaires, la revente des droits, la vente de biens ou services annexes ou dérivés. Étudiant cette modélisation, Philippe Bouquillion et Jacob Matthews (2010) ont souligné l'oubli de " la collecte de données de plus en plus précises sur les internautes et leurs usages »(p.19), qui 
Pour une approche de l'éducation critique aux médias par le décryptage des logiques politiques, économiques, idéologiques et éditoriales du numérique

constitue pourtant une ressource monnayable et une stratégie pour améliorer la valorisation des espaces publicitaires. L'économie des plateformes du Web est en effet présentée aujourd'hui comme dominée par les enjeux liés aux big data fondés sur le recueil des traces numériques. Ce fonctionnement repose en outre sur une intrusion systémique dans la vie privée des usagers, comme le montre Éric George (2011) à propos de Google.

C'est en ce sens qu'un partenariat entre un service public d'éducation et une entreprise comme Microsoft interroge ${ }^{5}$. II permet certes d'équiper les classes à moindres frais, mais acculture les apprenants très tôt à une certaine gamme d'outils portant le label d'une marque commerciale, favorisant leur intégration à une chaîne de travail qu'ils seront amenés à utiliser dans leur vie professionnelle et leur fidélisation à la marque dont l'école accroît la légitimité. De plus, le contrat permet à l'entreprise de viser un objectif stratégique parallèle, celui de mieux connaître les pratiques des "jeunes " pour les cibler en tant que consommateurs : le risque que représente la récolte des traces au sein des outils connectés est complètement occulté par les éléments de discours courants prônant la modernité, la nouveauté, l'ouverture et l'innovation apportées à l'école par le numérique.

Pour le fondateur d'Atari, Nolan Bushnell, le numérique constitue le remède miracle contre le «syndrome du prof ennuyeux " (cité dans Rastier, 2013, p. 72), vraie cause, selon lui, des problèmes d'attention chez les élèves. Une « pédagogie par projets ", visant des "compétences" et listant des objectifs échelonnés par grilles, accompagne en effet parfois l'introduction du numérique sur le terrain des pratiques pédagogiques. Comme le rappelle François Rastier (2013), la " pédagogie par objectifs" est portée par une idéologie managériale, née aux États-Unis avec l'essor du taylorisme, " [d]'où le succès de la notion de compétence, terme clé du secteur des ressources humaines, car elle permet de décliner les degrés de l'employabilité, comme si l'enfant n'était qu'un futur salarié » (p. 5).

On voit comment, en se plaçant dans le sillage des industries du numérique, l'école diffuse, au-delà des compétences

${ }^{5}$ Comme celui passé en France, voir 1.1. 
instrumentales, des postures pédagogiques en phase avec des discours managériaux. Elle transmet aussi des modalités de travail qui participent des formes de production de la valeur par les plateformes numériques. Le digital labor, travail numérique fourni par les usagers, convertit la participation en valeur monétisée par les plateformes. On fait ainsi appel aux digital workers pour améliorer le fonctionnement des plateformes, par l'ajout de listes de recommandations, de commentaires, de photos ou de vidéos, en échange de rémunérations très fragmentées, voire inexistantes. Cette conception du travail " en miettes" est dénoncée aujourd'hui par certains chercheurs comme une atteinte aux cadres sociaux du travail, renforçant la responsabilisation des travailleurs. Elle crée en outre une dépendance vis-à-vis de plateformes qui imposent sans négociation leurs conditions d'utilisation (Casilli et Cardon, 2015). L'école vient donc valider, au nom de la dimension coopérative et innovante, des formes de travail qui peuvent être analysées comme régressives sur le plan du droit social. Ici encore, une éducation critique au numérique peut passer par la prise de conscience de logiques de production invisibles pour l'utilisateur.

\subsection{Décoder les outils industriels de l'écriture numérique}

L'emprise de l'économie de l'attention (Goldhaber, 1997) se ressent jusque dans les modalités de l'écriture numérique. C'est pourquoi il est important d'interroger aussi la relation entre ces processus de création et d'éditorialisation et les dispositifs sociotechniques qui les soutiennent. Des chercheurs comme Yves Jeanneret (2016) observent depuis des années comment les outils numériques industriels tentent d'«enformer » les êtres en standardisant les pratiques d'expression. La forme du texte et de l'image, inséparable du sens qui émerge à partir du contenu, se trouve régie par les propositions de formatage des outils d'écriture.

L'hégémonie des industries du numérique se fonde sur des orientations stratégiques, qui nous privent de plus en plus des possibilités de stockage privé (suppression des lecteurs de CDROM, par exemple), et nous pousse vers le "nuage ", plus facilement exploitable par elles. Elle s'infiltre également dans 
Pour une approche de l'éducation critique aux médias par le décryptage des logiques politiques, économiques, idéologiques et éditoriales du numérique

les pratiques ordinaires, à travers les " petits détails " dans les logiciels, propriétaires courants. Les métaphores graphiques (pinceau, gomme, imprimante, dossier, ciseaux...) et les instructions verbales formulées d'avance («fichierenregistrer ") dans Word facilitent le dialogue avec la machine tout en occultant les calculs effectués sur les données des utilisateurs. Les pratiques culturelles, l'écriture, la photographie, la musique, dès lors qu'elles s'appuient sur des outils numériques, entrent en négociation avec les stratégies qui se trouvent incarnées dans les " détails » soutenant la structure éditoriale: structure qui, tout en promettant une facilitation d'accès aux pratiques, formate l'expression, donc la pensée, et occulte les multiples enjeux marchands et politiques qui la motivent.

Faut-il aller jusqu'à considérer les outils numériques industriels comme des "producteurs d'aliénation", dans le sens de Gilbert Simondon (2012, p. 339) ? Le décryptage critique de l'" architexte " des logiciels et des plateformes (formulaires, cadres, menus et barres d'outils qui à la fois facilitent et norment l'écriture numérique) nous paraît en tout cas constituer un enjeu important de l'éducation aux médias. À défaut de pouvoir toujours accéder à l'architexte (c'est notamment vrai pour les moteurs de recherche et les réseaux sociaux numériques), l'éducation aux médias devrait du moins donner aux apprenants les moyens de lire les traces que ces stratégies de formatage laissent dans leurs pratiques. Une analyse du design et des contenus de blogues de mode ou de beauté, par exemple (corpus très prisé par les adolescents et les jeunes adultes), peut montrer à quel point les auteurs écrivent en réalité sous la pression d'une double contrainte : contrainte exercée à la fois par les plateformes de blogues comme WordPress, qui, sous couvert de gratuité, uniformisent les pratiques d'expression et exploitent les contenus créatifs sans la moindre rémunération (voir la notion de digital labour exposée plus haut), et par les marques, qui commanditent grand nombre de contenus de façon ouverte ou occultée, orientant à leur tour la production des contenus. 


\section{Mettre au jour les ressorts idéologiques des utopies du numérique}

L'influence de l'idéologie techniciste dans le "culte » que nos sociétés accordent à la communication informatisée a été mise en évidence depuis l'ouvrage de Philippe Breton et Serge Proulx (1991). Sa critique est devenue un leitmotiv des études sur internet (Breton et Proulx, 1991 ; Cardon, 2010 ; Flichy, 2008 ; Proulx, 2015 ; Rebillard, 2007) sans pour autant figurer au cœur de ce qui serait une éducation critique aux médias. En France, l'Éducation nationale envisage l'éducation aux médias et à l'information comme centrée sur l'analyse des contenus, la recherche d'information, les démarches de validation de l'information et l'utilisation des outils technologiques, comme si les plateformes numériques elles-mêmes pouvaient être considérées comme un espace neutre. Leurs modalités de fonctionnement incarnent pourtant une gouvernance néolibérale qui s'appuie sur les ressorts d'utopies déjà expérimentées.

\subsection{Utopie d'une communication libre, porteuse de démocratie et d'égalité}

Les technologies de l'informatique ont réinvesti la promesse d'une liberté apportée par la communication, dont Pierre Musso (2002) a montré qu'elle remontait au moins au saint-simonisme qui prédisait déjà la démocratie et la suppression des classes et des inégalités par la communication et le développement des chemins de fer. Cette utopie progressiste et techniciste a connu des variantes depuis la cybernétique de Norbert Wiener et Marshall McLuhan. Fred Turner (2012) a montré son réinvestissement par la contre-culture californienne et le renouveau utopique qui a caractérisé les fondements de l'internet, surtout avant les années 2000. Investi par des personnalités ayant expérimenté le communalisme hippie pour y construire un monde nouveau, internet a été le support du rêve d'un exil dans un territoire libéré des contraintes, valorisant à la fois l'auto-organisation, la liberté et la coopération. Cette utopie serait matérialisée dans le design même des écrans, carrés pour reprendre la symbolique du jardin, dont Apple et son Macintosh seraient les "fruits défendus " (Frau-Meigs, 2011). 
Pour une approche de l'éducation critique aux médias par le décryptage des logiques politiques, économiques, idéologiques et éditoriales du numérique

Cette utopie imprègne encore aujourd'hui le marketing des acteurs numériques dominants (Google, Facebook, Amazon, Apple, Microsoft) et conforte certainement les discours productivistes centrant les politiques publiques sur la diffusion des équipements. Dans le cadre de l'école, l'utopie égalitariste pose cependant un souci que les chercheurs n'ont pas manqué de souligner depuis 15 ans. En 2001, dans un article devenu célèbre, "Digital Natives, Digital Immigrants », le journaliste Mark Prensky diagnostiquait un changement radical dans le rapport au savoir et au sérieux des nouvelles générations nées avec le numérique et préconisait de remettre en cause les pratiques pédagogiques traditionnelles. Dans sa lignée, Michel Serres (2012), en France, a popularisé l'idée que la «Petite Poucette », jeune "native » du numérique n'aurait pas besoin d'une éducation aux médias numériques parce qu'elle les maîtriserait déjà. Le parti pris est aussi idéologique que celui qui consiste à dépeindre l'élève devant son écran comme un "cyber-benêt», victime naïve des théories du complot et autres manipulations. Les nombreuses recherches sur le sujet montrent que, si le numérique peut être un facteur d'intégration et d'assimilation pour certains, le fait de pouvoir tirer bénéfice des potentialités de l'internet pour la recherche d'information, la construction de réseaux sociaux et le développement des carrières professionnelles n'a rien de mécanique, et que, si les inégalités sociales peuvent être reconfigurées par l'internet, les rapports aux TIC sont d'abord inscrits dans la matrice des rapports sociaux (Bourdeloie, 2012; Brotcorne et Valenduc, 2009 ; Hargittai, 2002 ; Jouët, 2000).

Le numérique génère en réalité une pression considérable sur les jeunes apprenants. Les représentations du numérique prônent son " potentiel émancipatoire » et font du non-usage une sorte de déficience sociale, comme le fait remarquer Rémy Rieffel (2014). Les recherches en milieu scolaire montrent que les compétences numériques réelles des élèves en matière de recherche de l'information sont très inégalement distribuées. C'est moins l'accès aux outils ou leur manipulation qui posent aujourd'hui problème que la capacité des apprenants à en extraire l'information et à verbaliser les difficultés liées à cette tâche.

La "classe inversée », parfois prônée comme un idéal pédagogique facilité par l'informatique connectée, en dehors 
des questions de traçage des pratiques et de leur exploitation marchande, risque donc surtout de creuser encore certaines inégalités: renvoyant l'acquisition des connaissances vers le " hors-classe ", elle néglige le fait que l'environnement familial des élèves n'est pas toujours propice à l'acquisition de connaissances ni à un usage expert de l'internet. L'accès des parents à l'internet dans le cadre du travail est en effet encore très différencié selon les catégories socioprofessionnelles et favorise des compétences très inégales. Les médiations parentales s'en ressentent nécessairement.

La connaissance du fonctionnement des industries culturelles permet de comprendre le discrédit jeté sur les adultes comme l'un de ses moteurs. Le fonctionnement du capitalisme repose sur un processus de destruction créatrice, comme l'a montré Schumpeter. Les jeunes générations jouent un rôle clé dans le capitalisme pour favoriser l'obsolescence des productions et le renouvellement des équipements. Cela explique notamment le rôle de la jeunesse dans les discours publicitaires depuis au moins les années 1930. Celle-ci joue un rôle de meneur dans l'adoption des innovations culturelles, entraînant le reste de la population et ringardisant les pratiques précédentes. Les industries du numérique sont plus centrées encore sur les pratiques des adolescents qu'elles scrutent et qui orientent la plupart de leurs stratégies de développement: importance de la communication visuelle, expressive, voire éphémère, comme pour rester au plus près du rythme pulsionnel de l'adolescence.

\subsection{Utopie de nouvelles formes de gouvernance, fondées sur la coopération}

Le numérique est porteur d'un projet politique ambivalent dont les sources se trouvent à la fois au fondement de l'imaginaire du réseau, de la contre-culture californienne et de sa rencontre avec le néolibéralisme. L'idéologie néolibérale promeut la perspective d'un individu rationnel, capable d'appliquer à toutes les sphères de sa vie une conduite dictée par la loi du marché, de "se penser comme une entreprise " (Dardot et Laval, 2009). Elle promeut un individu autonome, conforme au "nouvel esprit du capitalisme " (Boltanski et Chiappello, 1999), gouverné par des projets, résultats d'une 
Pour une approche de l'éducation critique aux médias par le décryptage des logiques politiques, économiques, idéologiques et éditoriales du numérique

" participation » volontaire à un ordre managérial consenti. Les hiérarchies et les relations de pouvoir sont effacées, laissant croire à leur disparition, comme à celle des inégalités de classe. Le néolibéralisme a ainsi récupéré les utopies politiques de progrès social, de développement personnel, d'autonomisation des individus et même de coopération, tout en organisant une compétition de tous contre tous. Sur les réseaux sociaux, les conditions d'une collaboration égalitaire et fructueuse ne sont pas remplies (Sennett, 2013). C'est au contraire la compétition des ego, la valorisation des personnalités non coopératives que mettent en scène les murs de Facebook, suscitant la curiosité, pour ne pas dire «l'espionnage » des autres, comme l'affirment eux-mêmes les adolescents (Jehel, 2016). Les communautés si utiles pour la valorisation des plateformes sont par ailleurs traversées de plus en plus par les logiques promotionnelles des annonceurs, qui souhaitent profiter de l'atmosphère de confiance que favorise la discussion entre pairs, entre amateurs, pour promouvoir leurs marques.

Dans le cadre de l'EMI, il serait particulièrement utile de construire un regard critique sur le discours " coopératif » des plateformes numériques et de transmettre des modalités de travail susceptibles de favoriser une réelle coopération entre les élèves. L'ampleur des phénomènes de harcèlement et des postures de retrait des adolescents sur les réseaux sociaux devrait y inciter. À titre d'exemple, les programmes de téléréalité et l'utilisation controversée du consentement pourraient permettre de créer un espace de réflexivité sur un type de programme particulièrement présent dans la culture médiatique des adolescents (Jehel, 2015).

\subsection{Utopie de l'instantanéité, de l'ubiquité, du culte de la vitesse}

Si le fonctionnement technique et économique d'internet ainsi que le rôle des plateformes dans la recherche et l'accès à l'information sont encore peu étudiés en classe, la presse fait partie des objets traditionnellement associés à l'éducation aux médias, mais elle est souvent abordée par la seule analyse des contenus. Le décryptage conjoint des logiques économiques, éditoriales et discursives de la presse en ligne permet pourtant d'interroger de façon exemplaire les relations entre pouvoir et 
savoir, "entre forme et force idéologique », comme le formulent Eleni Mitropoulou et Nicole Pignier (2014), relations qui structurent les pratiques d'expression et de réception sur les dispositifs numériques.

Les concepteurs de sites Web de presse sont guidés dans leurs pratiques par les possibilités de la technique, diverses représentations de l'usager, mais aussi par les enjeux industriels évoqués plus haut (collecte des données, buzz-feed, surveillance des pratiques dans l'objectif du profilage de l'usager). Les représentations de l'usager reposent parfois sur une connaissance réelle de ses pratiques et de ses attentes, mais plus souvent s'appuient sur des "allants de soi » à caractère idéologique, mobilisés pour justifier des stratégies marketing. Sur la page-écran, ces représentations et ces stratégies se matérialisent dans des fonctionnalités, des formes éditoriales et des figures discursives. Leur agencement guide les pratiques de réception par le public (Saemmer, 2015). Le récepteur, quant à lui, approche ces matérialisations avec ses propres représentations et attentes et, comme le montrent la sociologie des usages et les études de réception, se laisse plus ou moins guider par les modélisations de pratiques à l'écran. Une approche critique des médias numériques telle que nous la concevons étudie à la fois les représentations des pratiques de réception par les concepteurs et leurs motivations possibles, les fonctionnalités, les formes et les figures de la production médiatique qui reflètent ces représentations et les représentations qui guident le récepteur avant et pendant l'usage et la réception de cette production.

Par exemple, nous avons pu constater que la lecture sur support numérique est considérée par beaucoup de jeunes lecteurs comme forcément rapide et impatiente (Saemmer, 2015). Cette représentation reçoit "le flambeau de la vérité " (pour reprendre les termes de Pierce), parce qu'elle a été forgée par les discours d'accompagnement médiatiques et industriels, qui prônent le caractère inexorable de cette pratique dans un mode accéléré. Face à cet interprétant collectif collecté grâce à des études empiriques, il s'agit premièrement d'inciter les apprenants à se questionner sur les fondements idéologiques de ces représentations: lit-on rapidement sur dispositif numérique parce c'est «naturel » ou parce que le design de la page-écran prescrit une telle pression temporelle? 
Pour une approche de l'éducation critique aux médias par le décryptage des logiques politiques, économiques, idéologiques et éditoriales du numérique

Est-ce qu'on papillonne parce qu'on le décide, parce que le producteur le suggère par la forme et le contenu proposés ou parce que les contenus eux-mêmes sont si pauvres qu'ils ne valent pas la peine d'être lus de façon concentrée et réflexive? L'analyse de productions numériques débute ainsi par un travail d'introspection idéologique, qui met à nu les routines et les " allant de soi » des acteurs (apprenants et enseignants) lors de l'acte de réception.

Est ensuite analysé comment les formes éditoriales et les figures discursives d'un site de presse relancent et intensifient ces interprétants. En dehors des grandes " unités éditoriales » (Saemmer, 2016) structurant les pages-écrans des productions numériques (comme l'agencement des contenus en mosaïque de titres brefs et racoleurs, voire, par exemple, la " une " de liberation.frou la colonne intitulée "News 24/7 » sur le site Web de BFM TV ${ }^{6}$ répertoriant par ordre chronologique les dernières "brèves " de la journée), conçues pour faciliter le parcours rapide de la "une » d'un site de presse, une accélération des pratiques se trouve également encouragée par grand nombre d'hyperliens. L'un des enjeux de l'hyperlien dans la presse en ligne est en effet non pas de fournir des compléments d'information, comme le supposent la plupart des jeunes lecteurs que nous avons pu interroger, mais de favoriser le classement du site par les moteurs de recherche : si le texte du lien s'adresse au lecteur, son code s'adresse avant tout à Google. Un groupe de presse a par conséquent tout intérêt à encourager la pose de liens internes, notamment dans le content management system mis à disposition des journalistes pour saisir leurs textes et insérer leurs hyperliens - quitte à engager ainsi une "circulation circulaire de l'information" (Rebillard, 2006, 2011, à la suite de Bourdieu) qui transforme le lecteur en activateur mécanique de pages et le journaliste, en producteur de contenus buzz-feed (Saemmer, 2017).

La pression temporelle subie dans la production et la lecture d'un article de presse en ligne pourrait être mise en regard des exigences du capitalisme moderne qui transforme, depuis le $18^{\mathrm{e}}$ siècle, l'espace public en espace transparent favorisant la circulation rapide et le spectacle des marchandises (Sennett, 1973). La production est soumise à l'exigence de gagner du

${ }^{6}$ Chaîne de télévision d'information en continu. 
temps, comme l'avait si bien illustré Chaplin dans Les temps modernes (1936). Le gain de temps, l'obsession du chronomètre au cœur du dispositif tayloriste ont comme résultat une perte du sens, celui du travailleur à la chaîne, mais bientôt aussi de tous ceux qui sont assis à la chaîne de leur ordinateur. Pression temporelle vécue de façon aiguë par les cadres, soumis à une exigence d'« hyperformance ", au risque d'une " combustion de soi » (Aubert, 2006). On peut se demander si les signes de lassitude que certains adolescents manifestent maintenant vis-à-vis des réseaux sociaux ne relèvent pas déjà d'une nouvelle logique de travail qui n'a pas encore conscience d'elle-même et qui imprègne les activités numériques (Jehel, 2017).

\section{Favoriser les voies d'une expression citoyenne et émancipée}

\subsection{Les enjeux politiques et citoyens de l'accès à l'espace public}

La dimension économique et idéologique des industries du numérique ne doit pas occulter les enjeux politiques des espaces publics médiatiques en démocratie et le lien d'interdépendance mis en évidence par Habermas entre espaces publics médiatiques et démocratie, dans la sociogenèse de l'espace public bourgeois au $18^{\mathrm{e}}$ siècle. Ces enjeux nourrissent l'attente toujours vive d'une démocratisation de la décision politique par les dispositifs numériques (pétitions, blogues, réseaux sociaux). Même si les résistances des appareils institutionnels (partis politiques, administrations, pouvoirs publics) sont fortes, les dispositifs numériques sont porteurs de nouvelles formes de contre-démocratie, entendues comme promesse d'un renouvellement de la démocratie par la force qu'elles confèrent aux contre-pouvoirs (Rosanvallon, 2008), et favorisent la diffusion de discours militants, ceux des " médiactivistes " (Cardon et Granjon, 2013) permettant de renouveler l'espace public traditionnel. La structure relativement ouverte de l'internet permet également l'expression de publics " subalternes » (Fraser, 2001), dont la participation sur les autres espaces médiatiques est plus souvent minorée, voire stigmatisée. Le développement des plateformes lui-même sollicite chacun pour venir exposer ses "actualités" et 
Pour une approche de l'éducation critique aux médias par le décryptage des logiques politiques, économiques, idéologiques et éditoriales du numérique

participer à des échanges publics. La participation à un espace public est un moment essentiel pour chacun, afin de trouver une dignité et un sentiment d'existence plus grand parce qu'il est partagé avec d'autres (Arendt, 1958).

L'essor des plateformes s'est cependant accompagné en Europe d'une dérégulation profonde des espaces publics médiatiques. Le choix a été fait, au niveau de l'Union européenne, de diminuer le degré de la régulation publique en le remplaçant par un appel à l'« autorégulation » des acteurs économiques et des internautes. L'école, notamment avec I'EMI, doit endosser la responsabilité, dans ce contexte, de transmettre les repères de cette autorégulation à des adolescents qui, chaque jour, font l'expérience du chaos informationnel de l'internet, pour des raisons qui débordent largement l'impact de la propagande terroriste sur le Web. Ce pêle-mêle informationnel résulte pour une part du fonctionnement des plateformes, par l'intermédiaire de leurs algorithmes, mais aussi de l'absence de régulation des contenus qui y circulent. Pour les adolescents, le fil d'actualité se présente alors comme une succession d'informations hétérogènes, dont il leur est difficile de comprendre le sens et de connaître la source. Dans ce contexte confus, certains ont du mal à prendre conscience de la gravité de certaines informations, d'en évaluer la validité.

\subsection{Opacité du fonctionnement des plateformes et de leur moteur algorithmique, obstacle à l'émancipation citoyenne}

Si la recherche de l'information est l'une des activités les plus partagées par les adolescents (98\% des 12-17 ans, IFOP, 2013, France), la problématisation du dispositif lui-même et de ses caractéristiques est en revanche rarement mise en œuvre spontanément. Par exemple, l'équipement à domicile impose souvent Google comme unique page de lancement du navigateur (Cordier, 2015). L'influence du moteur sur le classement de l'information n'est pas thématisée, ou alors il l'est de façon problématique: "Quand je suis sur Google, je prends le premier résultat parce que c'est le premier qui vient, et puis ma mère, elle m'a dit de toujours prendre le premier ", explique un élève à Anne Cordier (2015). Une modification 
récente de l'algorithme de recherche de Google appliquée aux contenus de propagande en faveur de Daech montre à quel point l'entreprise connaît les pratiques de recherche de ses usagers et s'y adapte: faisant fi du principe de neutralité " mathématique " habituellement brandi pour justifier le classement des entrées, Google a décidé de faire remonter à la une des pages de résultats des vidéos montrant des victimes de Daech, parents désespérés et martyrs repentis. Certes, Google essaie ainsi d'assumer un rôle pédagogique dans le tri de l'information, réclamé par les usagers et les institutions, mais l'efficacité d'une telle mesure reste à prouver. En outre, cette mesure nous amène à nous interroger sur la validité des principes de classement de Google qui, jusqu'alors, avaient hiérarchisé les sites non pas selon leur sens, mais selon la quantité de liens entrants. Qu'en est-il de la prétendue " neutralité » des algorithmes de Google à partir du moment où l'entreprise commence à classer les sites selon des principes de sens ? Qu'en est-il de la promesse de transparence à partir du moment où de telles manipulations commencent à être mises en œuvre? Des questions de même nature se poseront d'ailleurs avec le développement d'outils de lutte contre les fausses nouvelles (dans le cadre de First Draft).

Ces dernières années, l'éducation à l'information et aux médias s'était donné comme principal objectif de former à la sélection de sources d'information pertinentes parmi les propositions faites par les moteurs de recherche. Si cet enjeu reste important à l'heure actuelle, les réseaux sociaux sont cependant de plus en plus utilisés pour s'informer. L'ordre d'apparition des messages sur le fil d'actualité de Facebook est construit par l'algorithme de la plateforme, EdgeRank. Reposant à l'origine sur trois critères (l'affinité, le poids différencié des contenus et la préférence pour les contenus les plus récents), l'algorithme intégrerait désormais " 100000 paramètres » (Le Monde, $1^{\text {er }}$ juillet 2014). Le travail de recommandation dont le fil d'actualité est le résultat vise à prédire les contenus susceptibles d'intéresser le plus l'internaute. Pour ce faire, il relève ses actions (like, clics, publications), celles de ses "amis", les like reçus, les interactions entre l'internaute et ses contacts, et fonctionne sur un modèle machine learning (intelligence artificielle). De nombreux chercheurs redoutent cette fermeture de l'information sur des choix passés, qui réduit l'horizon de leur possible 
Pour une approche de l'éducation critique aux médias par le décryptage des logiques politiques, économiques, idéologiques et éditoriales du numérique

évolution et de leur tolérance à l'altérité (Rouvroy, 2014). On peut également s'inquiéter de l'effet de dépossession du fonctionnement de leurs comptes par les internautes.

Le fil d'actualité, comme les échanges qui peuvent avoir lieu sur les réseaux sociaux, s'écarte, et de beaucoup, de la promesse de coopération et de bien-être. Ils sont vécus par un nombre grandissant d'adolescents comme des espaces de contrôle social étroit, risquant à tout moment de se transformer en insultes et en zone de non-droit (Jehel, 2016b).

Plutôt que d'affronter les théories " complotistes " les plus fantaisistes, la connaissance des règles de droit auxquelles sont en principe soumis les espaces médiatiques pourrait renforcer des pratiques citoyennes tout en les protégeant de leur violence. Le développement d'une connaissance de la déontologie journalistique peut favoriser, comme nous en faisons chaque année l'expérience, le basculement des jeunes dans des postures critiques. La déontologie n'est alors pas enseignée comme un espace fermé de règles, mais comme une trousse à outils permettant d'interroger le bien-fondé des démarches médiatiques.

\subsection{Promouvoir les pratiques de résistance}

Des calculs algorithmiques sont effectués à partir de chaque trace que l'utilisateur laisse sur l'internet par son passage par les dispositifs connectés - traces qui configurent également de plus en plus l'accès à l'information, donc la vision du monde qui en résulte. La " mise en scène de soi » à travers des selfies sur les réseaux sociaux n'est que la couche la plus visible et conscientisée d'une vaste "présence numérique ", alimentée de traitements algorithmiques à partir des like, des lectures et des recherches d'information, des écritures et des images publiées en ligne. Alors que l'école s'est beaucoup questionnée sur les éventuels dangers inhérents aux pratiques de mise en scène de soi sur les réseaux sociaux numériques, c'est aussi cette " présence numérique ", souvent occultée et enfouie, qu'il faut analyser de près : le fait qu'elle est "procédurale plutôt 
qu'expressive », comme le formule Louise Merzeau ${ }^{7}$, qu'elle est calculée à l'insu de l'utilisateur à partir des traces qu'il laisse au quotidien. Si cette " identité procédurale » suit donc l'utilisateur des dispositifs numériques comme une ombre, il s'agira d'une part, comme le formule Dominique Cardon, d'entrer dans les calculs et d'identifier leurs « visions du monde ${ }^{8}$ "; d'autre part, pour revenir aux propositions de Louise Merzeau, le sujet pourrait commencer à mettre en scène sa traçabilité, à documenter la captation des données en "surjouant » le jeu des algorithmes.

Le sujet établit sa présence numérique à travers les informations qu'il met à disposition, consciemment ou inconsciemment. À la surface de l'écran, c'est désormais le «profil » de l'utilisateur qui sert de boussole pour aller vers l'information, et non plus seulement les sites Web des médias classiques comme la presse et la télévision, ou les interfaces de saisie des moteurs de recherche. Avec les effets de renfermement que cela peut occasionner, pour ceux dont les réseaux de contacts sont très homogènes. Pour empêcher le monde de "se plier » d'une seule façon, Louise Merzeau recommande d'une part de changer régulièrement de plateforme ${ }^{9}$. D'autre part, elle avance qu'il faudrait considérer l'internet non plus comme le lieu d'une gestion, mais d'une marche, dans des bases de données qui sont transformées par nos pratiques et qui devraient nous inciter à inventer des « chorégraphies ». À travers la production, la gestion, l'éditorialisation et le partage de collections, d'albums, de chaînes vidéo, l'identité procédurale pourrait ainsi se reconnecter à une identité expressive.

Se forment aussi, au sein même des industries culturelles, des poches de résistance qui proposent des alternatives au buzz-feed et à l'accélération ambiants. L'hyperlien dans la presse en ligne par exemple, d'abord outil de buzz-feed,

\footnotetext{
${ }^{7}$ Louise Merzeau, intervention dans le cadre du séminaire «Pour une éducation critique aux médias en contexte numérique », Laboratoire Cemti, université Paris 8 , mars 2016.

${ }^{8}$ Dominique Cardon, intervention dans le cadre du séminaire «Pour une éducation critique aux médias en contexte numérique ", Laboratoire Cemti, université Paris 8 , avril 2016.

${ }^{9}$ Louise Merzeau, intervention dans le cadre du séminaire «Pour une éducation critique aux médias en contexte numérique », Laboratoire Cemti, université Paris 8 , mars 2016.
} 
Pour une approche de l'éducation critique aux médias par le décryptage des logiques politiques, économiques, idéologiques et éditoriales du numérique

continue à relier deux textes, et cette relation textuelle, en dépit ou malgré les enjeux marchands, peut faire émerger des significations qui ne relèvent pas que de la "circulation circulaire " du même. Certains hyperliens modélisent ainsi un lecteur qui ne cherche pas systématiquement la confirmation de ce qu'il sait déjà, ou même des informations simplement " complémentaires", mais qui accepte de mettre en perspective des opinions divergentes (pour des exemples, voir Saemmer, 2015, 2016) -lecteur qui n'entre pas dans le schéma courant de la lecture superficielle et papillonnante. Ces lignes argumentatives complexes permettent d'affirmer, contre l'injonction idéologique à l'accélération, un degré d'incertitude salutaire. Les études d'Anne Cordier en milieu scolaire ont montré que les adolescents s'attendent plutôt à ce que l'information en ligne réduise leurs incertitudes. L'un des élèves interrogés par la chercheuse souhaitait par exemple que Google enlève "toutes les choses fausses " de ses pages de résultats (Cordier, 2015). Dans ce contexte, il est cependant utile de rappeler que l'évitement de la polysémie est l'une des caractéristiques des totalitarismes. L'incertitude apportée par certaines caractéristiques de la textualité numérique est en réalité une chance pour l'éducation aux médias. Elle nécessite cependant la formation au décryptage de la rhétorique complexe des productions médiatiques et au débat d'idées raisonné à partir des points de vue mis à disposition des récepteurs.

Même si cette prise de conscience des usages et des pratiques de réception modélisés par les artefacts médiatiques en contexte numérique est un enjeu important pour favoriser l'émergence d'une attitude réflexive, l'objectif de l'EMI ne doit pas se réduire à la proposition d'outils de défense contre les effets idéologiques et les logiques marchandes. Se savoir, en tant que sujet utilisateur, sous le regard de diverses métriques et guidé par des structures de domination marchandes, politiques, éditoriales et discursives ouvre cependant déjà la voie aux possibilités pour y échapper : tel est en tout cas le point de vue que nous souhaitions défendre dans cette contribution. 


\section{Conclusion}

L'objectif de l'éducation critique aux médias en contexte numérique est, pour reprendre une formule d'Emmanuel Guez, de "saisir ce qui nous saisit ${ }^{10}$ ". La dépossession de soi par le numérique n'est pas une fatalité. La méthodologie que nous avons développée à partir de l'analyse des industries médiatiques et des logiques politiques, économiques, idéologiques et éditoriales propose des pistes pour une prise de conscience, par les apprenants comme par les enseignants, de la complexité des enjeux de l'EMI. Ce serait une première forme d'émancipation qui correspond au rôle humaniste et citoyen de l'école. Elle permettrait également de lever des résistances quand les enseignants ont le sentiment d'une instrumentalisation de l'école au profit de systèmes numériques de surveillance généralisée ou de modalités d'apprentissage par le numérique qui risquent de creuser des inégalités déjà trop présentes. Décoder l'emprise des modèles économiques sur les contenus médiatiques et numériques passe par l'analyse de l'impact de la maximisation de l'audience et du recueil des données personnelles, à la fois sur la production des artefacts et sur leur réception, à travers des dispositifs de cadrage, de formatage qui s'inscrivent aussi bien dans les discours de promotion que dans les textes, leur mise en image et en musique et dans les interfaces numériques. L'accès des amateurs et donc aussi des apprenants aux outils de production de contenu s'accompagne lui aussi du poids des contraintes induites par ces modèles de financement.

$\mathrm{Si}$ ces contraintes, malgré leur lourdeur, ne font pas disparaître l'enthousiasme et la croyance dans le progrès que représente le numérique, c'est que les industries médiatiques, au-delà des services rendus, reposent sur le déploiement d'utopies qui rendent le décryptage de leurs stratégies particulièrement complexe. Nous en avons rappelé quelquesunes: utopie d'une communication libre porteuse de démocratie et d'égalité; utopie de nouvelles formes de gouvernance plus horizontales fondées sur la coopération; utopie de l'instantanéité, de l'ubiquité et du culte de la vitesse.

\footnotetext{
${ }^{10}$ Emmanuel Guez, intervention dans le cadre du séminaire «Pour une éducation critique aux médias en contexte numérique », Laboratoire Cemti, université Paris 8 , avril 2016.
} 
Pour une approche de l'éducation critique aux médias par le décryptage des logiques politiques, économiques, idéologiques et éditoriales du numérique

Derrière elles, c'est le discours néolibéral et ses ambivalences qu'il devient urgent de mettre au jour, pour permettre à tous, sinon un libre consentement (devenu presque impossible du fait de l'inégalité des rapports de force), du moins une meilleure prise de conscience des doubles sens sur lesquels repose son succès. Certes, il est illusoire de croire qu'on peut amener les apprenants à " maîtriser » le numérique, dont certaines strates se soustraient obstinément au décodage critique. Mais décrypter le maillage complexe des strates qui constituent le design éditorial et les discours portés par les médias en contexte numérique, s'intéresser à ce que la matérialité des machines, des fonctionnements logiciels essaie de configurer comme visions du monde, donne déjà des clés pour comprendre et pour s'engager dans la construction de contrefigurations inventives, avec le numérique et en dehors.

\section{Références}

ADORNO T.W., 1954, «Quaterly of Film, Radio and Television », trad. de P. Beaud, J.-Y. Pidoux et G. Savary, "La télévision et les patterns de la culture de masse", Réseaux, vol. 9, n 44-45, 1990, pp. 225-242.

AGACINSKI D., F. BRUN, C. ISART, M. JAMES et S. POUTSLAJUS 2016, "L'École sous algorithmes », Terra Nova, <http://tnova.fr/etudes/l-ecole-sous-algorithmes>, dernière consultation le $1^{\mathrm{er}}$ juillet 2017.

Anderson C. et M. Wolff, 2010 " The web is dead. Long live the internet », Wired, <www.wired.com/2010/08/ff_webrip/>, dernière consultation le $1^{\text {er }}$ juillet 2017.

ARENDT H., 2008, Condition de l'homme moderne [1958], Paris, Calmann-Lévy.

ARENDT H., 1972, La Crise de la culture [1961], Paris, Gallimard.

AUBERT N., 2006, "Hyperformance et combustion de soi ", Études, t. 405, n 10, 339-351.

BARTHES R., 1964, "Rhétorique de l'image ", Communications, vol. 4, $\mathrm{n}^{\circ}$ 1, pp. 40-51.

BOLTANSKI L. et È. CHIAPELLO, 1999, Le Nouvel Esprit du capitalisme, Paris, Gallimard. 
BOUQUILLION P. et J.T. MATTHEWS, 2010, Le Web collaboratif. Mutations des industries de la culture et de la communication, Grenoble, PUG.

BOURDELOIE H., 2012, « L'Appropriation des dispositifs d'écriture numérique: translittératie et capitaux culturel et social », Études de communication, $\mathrm{n}^{\circ} 38$, pp. 23-36.

BOUTAUD J. et E. VERON, 2007, Sémiotique ouverte. Itinéraires sémiotiques en communication, Paris, HermèsLavoisier.

BRETON P. et S. PROULX, 1989, L'Explosion de la communication: la naissance d'une nouvelle idéologie, Paris, La Découverte.

BROTCORNE P. et G. VALENDUC, 2009, « Les compétences numériques et les inégalités dans les usages d'Internet », Les Cahiers du numérique, vol. 5, $\mathrm{n}^{\circ} 1$, pp. 45-68

CARDON D., 2015, À quoi rêvent les algorithmes. Nos vies à I'heure des big data, Paris, Seuil.

CARDON D. et F. GRANJON, 2013, Mediactivistes, Paris, Presses de Sciences Po.

CARDON D., 2010, La Démocratie Internet. Promesses et limites, Paris, Seuil.

CASILLI A. et D. CARDON, 2015, Qu'est-ce que le digital labor?, Paris, Ina.

CORDIER A., 2015, Grandir connectés. Les Adolescents et la recherche d'information, Caen, C\&F Éditions.

DARDOT P. et C. LAVAL, 2009, La Nouvelle Raison du monde. Essai sur la société néolibérale, Paris, La Découverte.

ECO U., 1992, Les Limites de l'interprétation, Paris, Grasset.

FRASER N., 2001, «Repenser la sphère publique: une contribution à la critique de la démocratie telle qu'elle existe réellement », Hermès, $n^{\circ} 21$, pp. 125-154.

FRAU-MEIGS D., 2011, Penser la société de l'écran. Dispositifs et usages, Paris, Presses Sorbonne Nouvelle.

FRAU-MEIGS D., M. LOICQ et P. BOUTIN, 2013, Politiques d'éducation aux médias et à l'information en France, ANR Translitt

Cost, 
Pour une approche de l'éducation critique aux médias par le décryptage des logiques politiques, économiques, idéologiques et éditoriales du numérique

$<$ www.enjeuxemedias.org/IMG/pdf/FRANCE_rapport_2014.p df>, dernière consultation le $1^{\text {er }}$ juillet 2017.

GEORGE É., 2011, «L'intrusion de Google dans la vie privée, au cœur des mutations du capitalisme », Terminal, $\mathrm{n}^{\circ} 108-$ 109, pp. 95-112.

GONNET J., 2001, Éducation aux médias. Les controverses fécondes, Paris, Hachette.

HARGITTAI E., 2002, "Second-Level Digital Divide: Differences in People's Online Skills », First Monday, vol. 7, $\mathrm{n}^{\circ} 4$, <http://firstmonday.org/ojs/index.php/fm/article/view/942 /864>, dernière consultation le 21 août 2017.

JEANNERET Y., 2016, 25 novembre, "Penser le programme et la prescription au sein de l'économie scriptuaire ", intervention dans le cadre du séminaire «Pour une éducation aux médias en contexte numérique " organisé par $\mathrm{S}$. Jehel et A. Saemmer pour le laboratoire Cemti.

JEANNERET Y. et E. SOUCHIER, 1999, "Pour une poétique de l'écrit d'écran », Xoana, n 6, pp. 97-107.

JEHEL S., 2017, "Actes des travaux du comité de recherche Sociologie de la communication", dans Congrès AISLF Montréal, du 5 au 7 juillet 2016, Montréal (Canada), pp. 3956.

JEHEL S., 2015, «L'éducation aux médias comme démarche participative et heuristique. Analyser les modalités de distanciation face aux programmes de téléréalité », dans M. Loicq et F. Rio (dir.), Les jeunes : acteurs des médias. Participation et accompagnement, Paris, Centre d'études sur les jeunes et les médias, pp. 119-130.

JEHEL S., 2016, "La Banalisation du harcèlement sur les réseaux sociaux numériques », Vers l'éducation nouvelle, $\mathrm{n}^{\circ}$ 561, pp. 53-65.

JEHEL S. et C. MAGIS, 2016, "Que peut l'éducation aux médias face aux discours discriminatoires? Difficultés pédagogiques et enjeux théoriques? », dans $\mathrm{S}$. Jehel et L. Corroy (dir.), Stéréotypes, discriminations et éducation aux médias, Paris, L’Harmattan, pp. 157-182.

JOUËT J., 2000, «Retour critique sur la sociologie des usages », Réseaux, n 100, vol. 18, pp. 486-521. 
MAIRESSE F. et F. ROCHELANDET, 2015, Économie des arts et de la culture, Paris, Armand Colin.

MARTY R., "Qu'est-ce qu'un interprétant? ", $<$ http://perso.numericable.fr/robert.marty/semiotique/s039.ht $\mathrm{m}>$, dernière consultation le $1^{\mathrm{er}}$ juilet 2017.

MITROPOULOU, E. et N. PIGNIER, 2014, Former ou formater? Les enjeux de l'éducation aux médias, Limoges, Solilang.

MOEGLIN P., 2015, "Quand éduquer devient une industrie ", Revue Projet, ${ }^{\circ} 345$, pp. 62-71.

MORIN E., 1961, «L'Industrie culturelle », Communications, $\mathrm{n}^{\circ} 1$, pp. 38-59.

MUSSO P., 2002, "L'économie symbolique de la société d'information », Revue européenne des sciences sociales, vol. XL, n 123, pp. 9-13.

OCDE, 2015, Connectés pour apprendre? Les élèves et les nouvelles technologies. Principaux résultats (rapport Pisa).

PIERCE C. S., 1931-1958, Collected Papers of C. S. Pierce, Cambridge, Belknap Press Harvard.

PRENSKY M., 2001, « Digital Natives, Digital Immigrants », On the Horizon, NCB University Press, vol. $9 \mathrm{n}^{\circ} 5$.

PROULX S., 2015, « Usages participatifs des technologies et désir d'émancipation: une articulation fragile et paradoxale », Communiquer, n 13, pp. 67-77.

RASTIER F., 2013, Apprendre pour transmettre. L'éducation contre l'idéologie managériale, Paris, PUF.

REBILLARD, F., 2011, "Modèles socioéconomiques du journalisme en ligne et possibilités d'une information diversifiée ", Les Enjeux de l'information et de la communication, $n^{\circ} 12 / 3$, pp. 81-95.

REBILLARD F., 2007, Le Web 2.0. en perspective. Une analyse socio-économique de l'internet, Paris, L'Harmattan.

REBILLARD, F., 2006, " Du traitement de l'information à son retraitement », Réseaux, n 137, pp. 29-68.

RIEFFEL R., 2014, Révolution numérique, révolution culturelle?, Paris, Gallimard. 
Pour une approche de l'éducation critique aux médias par le décryptage des logiques politiques, économiques, idéologiques et éditoriales du numérique

RIEFFEL R., 2005, Que sont les médias ? Paris, Gallimard.

ROSANVALLON P., 2008, La Légitimité démocratique, Paris, Seuil.

ROUVROY A., 2014, "Des données sans personne: le fétichisme de la donnée à caractère personnel à l'épreuve de l'idéologie des Big Data ", contribution en marge de l'étude annuelle du Conseil d'État, Le Numérique et les droits fondamentaux, pp. 407-434.

SAEMMER A., 2017, "Interpréter l'hyperlien en contexte pédagogique: éléments d'une sémiotique sociale », Le Français aujourd'hui, $\mathrm{n}^{\circ}$ 196, pp. 25-34.

SAEMMER A., 2016, "Sémiotique critique du discours hypertextualis ", Semen, $n^{\circ} 42$, pp. 135-156.

SAEMMER A., 2015, Rhétorique du texte numérique : figures de la lecture, anticipations de pratiques, Lyon, Presses de l'Enssib.

SCHOLTZ T. (dir.), 2012, Digital Labor: The Internet as Playground and Factory, New York, Routledge.

SENNETT R., 2013, Ensemble. Pour une éthique de la coopération, Paris, Albin Michel.

SERRES M., 2012, Petite Poucette, Paris, Le Pommier.

SIMONDON G., 2012, Du mode d'existence des objets techniques [1958], Paris, Aubier.

TURNER F., 2012, Aux sources de l'utopie numérique. De la contre-culture à la cyberculture, Stewart Brand un homme d'influence, Caen, C\&F Éditions. 